\title{
Contribution of Extracellular Matrix Component Landscapes in the Adult Subventricular Zone to the Positioning of Neural Stem/Progenitor Cells
}

\author{
Hyun Jung Kim ${ }^{1,2 \dagger}$, Eunsoo Lee ${ }^{1,3 \dagger}$, Myungwoo Nam ${ }^{1}$, Jae Kwon Chung ${ }^{1}$, Sunghoon Joo ${ }^{4}$, \\ Yoonkey $\mathrm{Nam}^{4}$ and Woong Sun ${ }^{1 *}$ \\ ${ }^{1}$ Department of Anatomy, College of Medicine, Korea University, Seoul 02841, ${ }^{2}$ Graduate School of Medical Science and \\ Engineering, Korea Advanced Institute of Science and Technology (KAIST), Daejon 34141, ${ }^{3}$ Fluorescence Core Imaging \\ Center, Ewha Womans University, Seoul 03760, ${ }^{4}$ Department of Bio and Brain Engineering, Korea Advanced Institute of \\ Science and Technology (KAIST), Daejeon 34141, Korea
}

\begin{abstract}
Neurogenesis persists in restricted regions of the adult brain, including the subventricular zone (SVZ). Adult neural stem cells (NSCs) in the SVZ proliferate and give rise to new neurons and glial cells depending on intrinsic and environmental cues. Among the multiple factors that contribute to the chemical, physical, and mechanical components of the neurogenic niche, we focused on the composition of the extracellular matrix (ECM) of vasculature and fractones in the SVZ. The SVZ consists of ECM-rich blood vessels and fractones during development and adulthood, and adult neural stem/progenitor cells (NS/PCs) preferentially attach to the laminin-rich basal lamina. To examine the ECM preference of adult NS/PCs, we designed a competition assay using cell micropatterning. Although both laminin and collagen type IV, which are the main components of basal lamina, act as physical scaffolds, adult NS/PCs preferred to adhere to laminin over collagen type IV. Interestingly, the ECM preference of adult NS/ PCs could be manipulated by chemokines such as stromal-derived factor 1 (SDF1) and a6 integrin. As SDF1 re-routes NSCs and their progenitors toward the injury site after brain damage, these results suggest that the alteration in ECM preferences may provide a molecular basis for contextdependent NS/PC positioning.
\end{abstract}

Key words: ECM, Basal lamina, Fractone, Subventricular zone, Neural stem/progenitor cell

\section{INTRODUCTION}

Neurogenesis continues to occur in restricted areas of the brain throughout life, which includes the subventricular zone (SVZ), subcallosal zone (SCZ), and the dentate gyrus of the hippocampus [1-5]. These adult neurogenic regions are believed to have unique neural stem cell (NSC) niches which are in close proximity

Submitted May 3, 2021, Revised August 11, 2021, Accepted August 28, 2021

* To whom correspondence should be addressed. TEL: 82-2-2286-1404, FAX: 82-2-929-5696 e-mail:woongsun@korea.ac.kr

These authors contributed equally to this work. to blood vessels [6-8]. NSCs and transit-amplifying cells reside adjacent to or near the blood vessels at sites lacking pericyte coverage and astrocyte endfeet [7-9]. In addition to providing nests for NSCs, blood vessels appear to act as a scaffold for the migration of adult SVZ cells to the olfactory bulb through the rostral migratory stream $[10,11]$ and toward injured areas after stroke [12-14]. In aging or diseased niches, vasculature deteriorates with a reduction in blood flow, followed by a decline in the neurogenic potential of NSCs and cognition $[15,16]$.

Several humoral factors such as Wnt, Shh, and TGF- $\beta$ are released from endothelial cells $[16,17]$, and other vascular-derived factors such as stromal-derived factor 1 (SDF1) and Ang1 promote neuroblast proliferation and survival [18]. Aside from these vesselderived humoral factors, vessels also provide the extracellular ma- 
trix (ECM)-based beds for neural stem/progenitor cells (NS/PCs). The ECM is ubiquitously found in the neurogenic niche, and NS/ PCs interact with the ECM at critical phases of each cell's life, such as migration [19]. Another unique ECM-rich structure found in the neurogenic niche is the fractones. Fractones are distributed on the apical surface of ependymal cells at the lateral ventricle, providing anchoring spots to the apical endfeet of NSCs as well as acting as reservoirs for humoral factors [20-23]. Although the origin and biological function of fractones are yet controversial $[21,24-$ 26], it has been widely believed that fractones are emerged from capillaries as thin stem, a conclusion supported by high-resolution imaging and similarities between the molecular compositions of fractones and the basal lamina [20,24-30]. ECM proteins in these structures regulate cell behavior via interactions with cell surface receptors, thereby mediating mechanical stress and serving as a reservoir for growth factors. For instance, SVZ progenitors express the laminin receptor $\alpha 6 \beta 1$ integrin, which mediates homing to the laminin-rich basal lamina of vessels in the SVZ [24]. Genetic ablation of integrin $\beta 1$ resulted in reduced neuronal progenitor proliferation and impaired cell migration on different ECM substrates $[26,31,32]$.

Collectively, these results suggest that the neurogenic niche is organized with specific ECM landscapes composed of basal lamina and fractones. While the basal lamina and fractones are complex mixtures of various ECM molecules, understanding the functional importance of each ECM protein within the complex mixtures is in its infancy. To address this issue, we explored the differential distribution of two major ECM proteins, laminin and collagen type IV, within neurogenic niches. Furthermore, using a micro-contact printing-based competition assay, we quantified NSC behaviors according to different ECM proteins as a simplified version of vascular ECM composition in vitro to monitor the behavior of adult NS/PCs.

\section{MATERIALS AND METHODS}

\section{Animals and neurosphere culture}

C57BL/6 mice at E18.5, P0, P7, and adulthood (8 9 weeks old) were used for this study (Orient Bio Inc., Seongnam, Korea). Neurosphere cultures were established from the anterior subventricular zone (SVZ) of adult mice as described [5]. Brains were removed and cut into thick coronal brain slices using a brain matrix. Freshly isolated SVZ tissues were first digested with $0.8 \%$ papain (Worthington) and $0.08 \%$ dispase II (Roche Applied Science) in Hank's Balanced Salt Solution (HBSS), followed by 45 min of incubation in $37^{\circ} \mathrm{C}$ water bath. Dissociated cells were plated onto an ultra-low attachment six-well plate for generating neurospheres in
DMEM/F12 media supplemented with 1\% N2, 2\% B27 and 1\% antibiotic-antimycotic (Invitrogen). Neurospheres were maintained by the daily addition of $20 \mathrm{ng} / \mathrm{ml}$ epidermal growth factor (EGF) and basic fibroblast growth factor (bFGF). At days in vitro (DIV) 8 , primary neurospheres were dissociated with Accutase (Innovative Cell Technologies), and multipotent progenitor cells were maintained as neurospheres by subsequent subculture. All experiments were carried out in accordance with the ethical guidelines of Korea University and with the approval of the Animal Care and Use Committee of Korea University.

\section{Tissue preparation and immunolabelings}

Mice were anesthetized and perfused with saline, and the SVZ region was dissected under a light microscope [33]. These wholemounts were fixed overnight in 4\% paraformaldehyde (PFA) with $0.1 \%$ Triton $\mathrm{X}-100$ at $4{ }^{\circ} \mathrm{C}$, washed three times with PBS, and blocked with $10 \%$ normal donkey serum albumin and $2 \%$ Triton X-100 in PBS for 1 day. For 3D imaging, some tissues were cleared by the ACT-PRESTO method as previously reported [34]. Briefly, brain samples were fixed in $4 \%$ PFA overnight at $4{ }^{\circ} \mathrm{C}$. Fixed samples were incubated in hydrogel monomer solution A4P0 (4\% acrylamide in $1 \mathrm{X}$ PBS) supplemented with $0.25 \%$ photoinitiator 2,2'-Azobis[2-(2-imidazolin-2-yl)propane] dihydrochloride (Wako Pure Chemical) overnight at $4{ }^{\circ} \mathrm{C}$. Hydrogel-infused samples were degassed for $2 \mathrm{~min}$ and polymerized for $2 \sim 3 \mathrm{~h}$ at $37^{\circ} \mathrm{C}$. Brain samples were washed in $0.1 \mathrm{X}$ PBS before tissue clearing. The polymerized samples were transferred into a cassette for an electrophoretic tissue clearing (ETC) chamber (X-CLARITY, Logos Biosystems), and the cassette was placed inside the chamber, which contained 4\% SDS and $200 \mathrm{mM}$ boric acid in $\mathrm{H} 2 \mathrm{O}$ (pH 8.5). The ETC was executed for $2 \mathrm{~h}$, and cleared samples were washed in $0.1 \mathrm{X}$ PBS for 1 day at room temperature with gentle shaking to remove the remaining SDS.

The normal or ACT-cleared tissues were incubated in the following primary antibodies for 2 days at $4{ }^{\circ} \mathrm{C}$ or 1 day at $37^{\circ} \mathrm{C}$ with gentle shaking : rabbit anti-LAMA1(laminin subunit a1) (1:500; Sigma), chick anti-laminin (1:1,000; Abcam), mouse anti-S100 $\beta$ (1:500; Sigma), goat anti- $\gamma$-tubulin (1:500; Santa Cruz), mouse anti- $\beta$-catenin (1:500; BD Bioscience), mouse anti-acetylated tubulin (1:500; Sigma), goat anti-collagen type IV (1:500; Millipore), rabbit anti-collagen type IV (1:1,000; Abcam), goat anti-collagen type IV (1:500; Millipore), or rabbit anti-epidermal growth factor receptor (EGFR) (1:500; Abcam) in 0.1X PBS containing 6\% bovine serum albumin (BSA), $0.1 \%$ Triton X-100, and 0.01\% sodium azide) with gentle shaking. The stained samples were washed with $0.1 \mathrm{X}$ PBS several times and then stained with fluorescently conjugated secondary antibody (1:500 dilution) for 1 day at $37^{\circ} \mathrm{C}$. The 
stained samples were then washed with 0.1X PBS. After incubation with appropriate secondary antibodies for 2 days, the wholemounts were trimmed and mounted onto glass slides for confocal imaging.

\section{Imaging and quantification of vascular ECM ratio}

Fluorescence images and initial sample visualization were performed using a conventional confocal microscope LSM700 and LSM880 Airyscan (Zeiss, Germany) at the Ewha Fluorescence Core Imaging Center. Image 3D-reconstruction was performed using the IMARIS imaging software (Bitplane, USA). To characterize the ECM signature of blood vessels in the SVZ and cortex, we used ImageJ to measure fluorescent signal intensities which labels collagen type IV and laminin. Grid $(1 \times 1 \mu \mathrm{m})$ was overlaid on the image, and a grid containing a single microvessel was marked as a region of interest (ROI). More than 20 ROIs were marked in each image, and signal intensity of red or green fluorescence was quantified after split into red and green images, respectively. In the scatter plot, each dot represented each quantification from the samesized area contained red and green signals from images of each brain region (Fig. 1F).

\section{Cell competition assay using micro-contact printing}

For the stripe assay, a laminin-stripe pattern (line width: $30 \mu \mathrm{m}$, spacing: $30 \mu \mathrm{m}$ ) was printed on a polydimethylsiloxane (PDMS) microstamp [35]. A PDMS stamp was fabricated using a soft lithographic technique and coated with a 10\% SDS solution to enhance protein loading on the surface of the stamp. A mixture of Cy3-conjugated immunoglobulin (1:500) and micro-printing substrates such as Poly-L-ornithine (PLO; $0.01 \%$, Sigma), collagen type IV (20 ng/ml; Sigma), and laminin isolated from EngelbrethHolm-Swarm murine sarcoma basement membrane $(20 \mathrm{ng} / \mathrm{ml}$; Invitrogen) were loaded onto the SDS-coated micro-stamp for $1 \mathrm{~h}$, while the coverslip was coated with one of the other substrates at $37^{\circ} \mathrm{C}$. The excess protein solution was removed by compressed air, and the coated PDMS stamp was immediately placed on a coated coverslip. A constant pressure (50 g) was applied to the stamp to transfer the protein from the stamp to the coverslip. Next, SVZderived progenitors were seeded at a density of $4 \times 10^{4}$ cells $/ \mathrm{mL}$ and incubated overnight at $37^{\circ} \mathrm{C}$. For antibody treatment, cultured cells were pre-incubated with anti-integrin a6/CD49f antibody (1:100, Novus Biologicals) for $1 \mathrm{~h}$ at $37^{\circ} \mathrm{C}$ prior to a micropattern assay. Pretreated cells were seeded onto a micropattern with serum-free medium containing mouse recombinant SDF1 (150 ng/ml; Peprotech).

\section{Immunostaining and image analysis}

Live cells were stained with Celltracker Green CMFDA ( $5 \mu \mathrm{M}$; Invitrogen) for $30 \mathrm{~min}$ and incubated in serum-free medium for 30 min. Cells were fixed with 4\% PFA and stained with DAPI. Samples were mounted onto a coverglass for confocal imaging (Zeiss LSM700). The results are representative of at least three independent experiments, and cells were counted from four images $(640 \mu \mathrm{m} \times 640 \mu \mathrm{m}$ in total) for each experiment. For the positioning of nuclei, the numbers of cells with their nuclei on/ off the micropattern were counted in every image. For the process alignment, the number of cells with their processes on pattern was counted, which suggest that the neurites recognize the micropattern as they grow in a parallel manner.

The statistical significance was calculated by Student's t test (twotailed) or analysis of variance (ANOVA) (least significant difference (LSD) for post hoc) for continuous variables. All statistical analyses were performed using SPSS software version 23.0 (IBM SPSS Inc.). All values are presented as mean and standard deviation, and $\mathrm{p} \leq 0.05$ was considered statistically significant. The linear regression of dot plot was performed using Prism 5 (GraphPad Software).

\section{RESULTS}

\section{ECM landscape in the SVZ}

The neurogenic region is known to have a specialized ECM architecture for the proliferation and maintenance of stem cells [7]. As previously reported, the SVZ contains an extensive vascular plexus and laminin-rich fractone bulbs on the ventricular surface (Fig. 1A). The fractones in the lateral ventricle were spherical and evenly distributed, whereas the shape and organization of fractones were less uniform in the third ventricle and absent in the fourth ventricle. The structural organization of SVZ was also confirmed by volume imaging using a tissue-clearing method (Fig. $1 B$, Supplementary video 1). Blood vessels exist in the SVZ at a depth of $\sim 100 \mu \mathrm{m}$, whereas speckle-shaped fractones were highly enriched at the surface of the ependymal layer. The en face view of the ventricular surface revealed that the spherical deposits were mainly located between ependymal cells at cell-cell contacts, and often at the center of pinwheel structures (Fig. 1C). Compared to adjacent blood vessels, these fractones were predominantly labeled with laminin, indicating that their ECM compositions were distinct from that of the nearby blood vessels (Fig. 1D).

The basal lamina of blood vessels is another major location for the presence of the ECM in the brain. The vascular plexus of the SVZ is made up of planar blood vessels, which extend long distances before branching into smaller vessels, while non- 


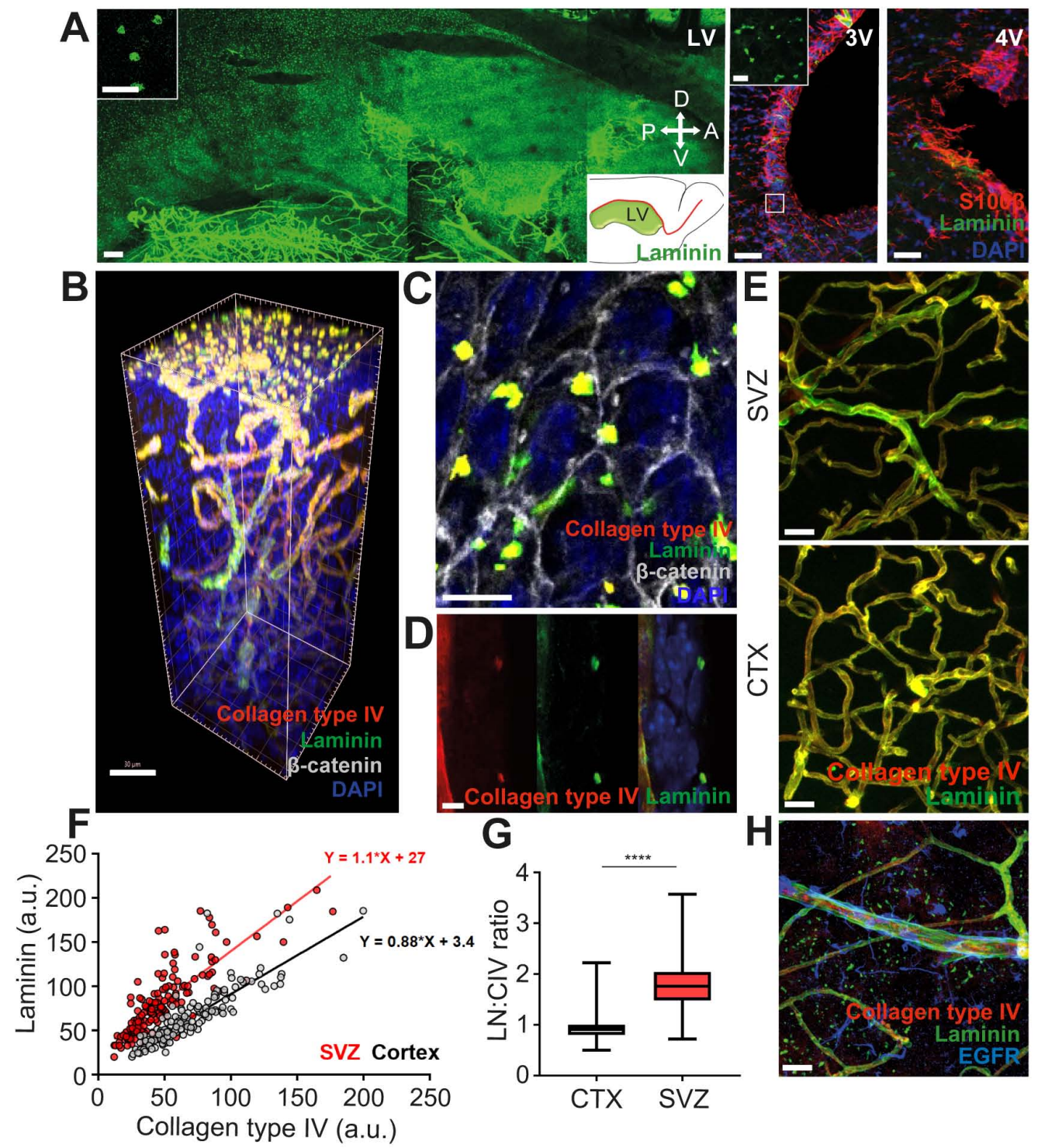

Fig. 1. Distribution of laminin and collagen type IV in the SVZ of an adult mouse brain. (A) Numerous speckle-shaped fractones are located on the ependymal layer in the lateral ventricle and $3^{\text {rd }}$ ventricle, with the exception of the $4^{\text {th }}$ ventricle. Speckle-shaped fractones are stained with laminin (green), and ependymal cells are stained with S100ß (red). Scale bar, $100 \mu \mathrm{m}$. Scale bar in the inset $10 \mu \mathrm{m}$. A, anterior; P, posterior; D, dorsal; V, ventral. (B) A 3D view of the SVZ region in a whole-mount preparation of the adult mouse brain. Scale bar, $30 \mu \mathrm{m}$. High magnified image of speckle-shaped fractones in the lateral ventricle ependymal layer whole-mount view (C), coronal sectioned view (D). Scale bar, $10 \mu \mathrm{m}$. (E) Comparison of vascular architectures in the SVZ and cortex using the z-projection. Scale bar, $20 \mu \mathrm{m}$ (F) A dot plot showing signal intensities of laminin and collagen type IV in the microvessels of SVZ and cortex. The best-fit linear regression line for SVZ is in red, and cortex in black. The intercepts and the slopes are presented for each brain region $(\mathrm{F}=12, \mathrm{p}=0.0005)$. ( $\mathrm{G})$ A boxplot showing the ratios between laminin $(\mathrm{LN})$ and collagen type IV (CIV) in blood vessels of SVZ and cortex. ${ }^{* * * *} \mathrm{p}<0.0001$. (H) Laminin and collagen type IV are distributed in different patterns along the branches of the vascular tree in the anterior and ventral parts of the SVZ. Transit-amplifying cells stained with EGFR (blue) are located in close proximity to laminin-rich blood vessels. Scale bar, $50 \mu \mathrm{m}$. SVZ, subventricular zone; EGFR, epidermal growth factor receptor.

neurogenic regions such as the cortex consist of tortuous vessels with multiple branches (Fig. 1E). When vessels were labeled with two main components of basal lamina, laminin and collagen type IV, we observed strong laminin expression in the blood vessels of SVZ, compared to that of cortex. Interestingly, branches sprouting from large vessels showed a relatively lower expression of laminin (Fig. 1E), indicating that the distribution of vascular ECM proteins, especially laminin content in the basal lamina, may act as a signaling cue for cell adherence and proliferation in the SVZ. Quantification of the intensity ratio of two ECM proteins on microvessels of the regions confirmed that laminin:collagen type IV ratio was significantly higher in the SVZ microvessels (Fig. 1F, 1G). 


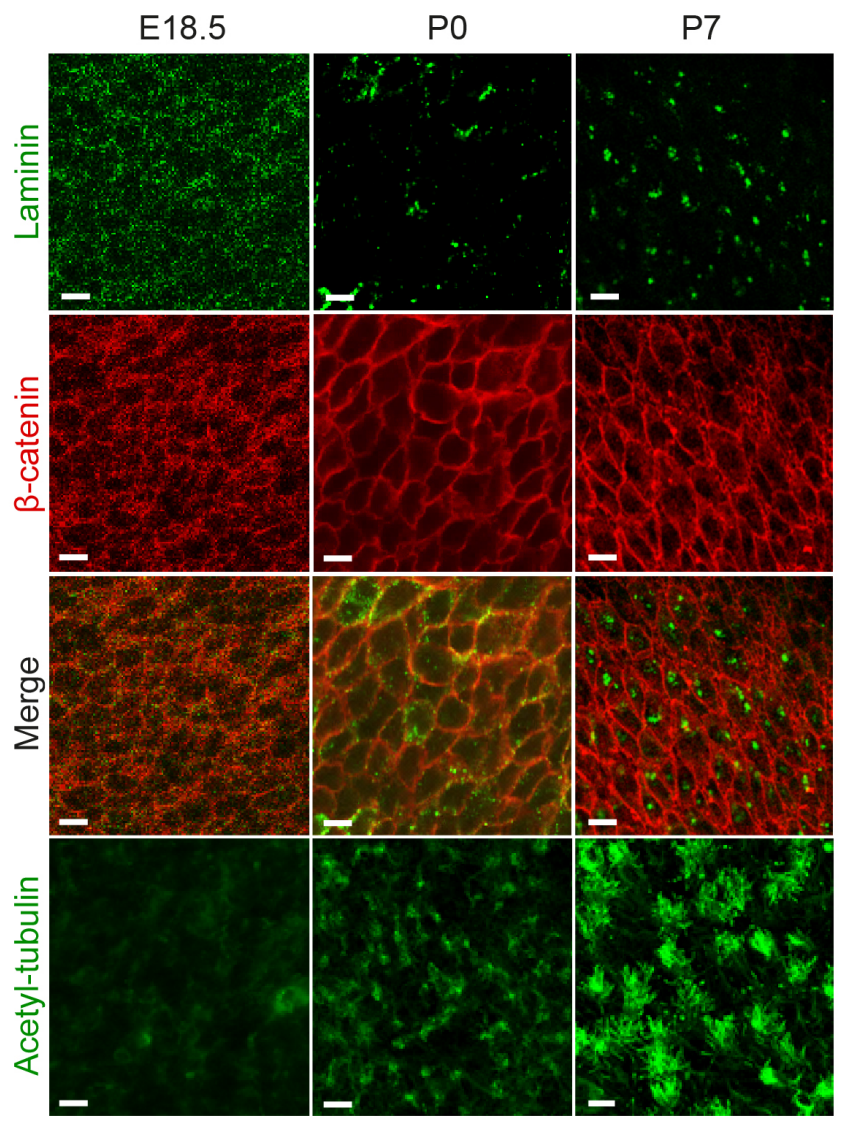

Fig. 2. Development of fractones in the ependymal layer. Confocal microscopy analysis of fractones at different stages of development; from left to right: E18.5, P0, and P7. Confocal images of lateral wall whole mounts from E18.5, P0 and P7 wild-type mice stained for laminin (green, top), $\beta$-catenin (red) and acetylated tubulin (green, bottom). Scale bar, $10 \mu \mathrm{m}$.

Several studies have reported that NSCs reside closer to the vascular plexus for spatial cues and regulatory signals $[7,24]$. To observe the influence of laminin heterogeneity on cell proliferation, we analyzed the localization of transit-amplifying cells labeled with EGFR (Fig. 1H). The majority of cells were located on or near the laminin-rich, large blood vessels, whereas fewer cells were found in close proximity to laminin-poor vessels. Accordingly, our findings also showed that laminin in the basal lamina structure plays a role in the cell adhesion of adult NS/PCs.

\section{Development of fractone bulbs and their association to cilia}

Although fractones were first described as a part of the endothelial basal lamina in the SVZ [24], the origin of fractones remains controversial $[21,25,26,36]$. Thus, we further investigated the organization and localization of fractones during brain development (Fig. 2). The laminin-rich small puncta were scattered on the surface ependymal layer during the embryonic stage (E18.5). During the remodeling of the ependymal layer, nearby fractones appeared to be joined together to form larger clusters at birth. At postnatal day 7 , the fractones became larger and were mainly located in the middle of the cell surface, but were not aligned with cell-cell contact sites as in the adult brain. This clustering and repositioning of fractones at the cell-cell contact sites were closely associated with the emergence of multiple cilia on the ependymal cell surface, suggesting the active role of cilia in fractone remodeling during these periods.

\section{ECM preference of adult NS/PCs}

To determine the adhesive effect of the ECM, we used a micropattern-based competition assay, which can simplify the complex nature of the ECM within the in vivo NSC microenvironment (Fig. 3A). Adhesive components of one of polyornitine (PLO), collagen type IV, and laminin were first coated on the surface of the glass coverslip, and a stripe-patterned ( $30 \mu \mathrm{m}$ wide) microstamp loaded with other adhesive components was stamped onto the pre-coated surface. After seeding of SVZ-derived progenitors, we measured the cellular localization with nuclei positioning and process alignment on micropattern. Since substrate composition and topography is known to affect neural progenitor alignment and cell morphology $[37,38]$, we used these parameters to determine whether the neurites recognize the overlaid ECM as they grow in a parallel manner. In this assay, the majority of cells adhered to the patterned laminin substrates overlaid on top of the PLO or collagen type IV surfaces (Fig. 3B). On the contrary, PLO patterned on the laminin resulted in a near chance value (50\%) distribution of cell bodies and the processes, and collagen type IV on laminin surfaces repelled the cell bodies and processes to the collagen-negative area (Fig. 3D, 3E). Since collagen type IV was overlaid on the laminincoated surface, these results suggest that collagen type IV inhibited the adhesive role of laminin in NS/PCs in the SVZ.

\section{Perturbation of ECM preference by blockage of $\alpha 6$ integrin signaling}

To understand how one ECM molecule can perturb the adhesive signal of other ECM molecules, we used the chemokine SDF1 and a specific blocking antibody of integrin to modulate ECM-directed cell behavior. The release of SDF1 enables blood vessels to act as a scaffold for the migration of SVZ progenitors toward injured areas after stroke [12-14], by the upregulation of EGFR and a6 integrin in the activated SVZ progenitors [39]. Thus, we hypothesized that SDF1 treatment might be important for migration along the vessels and release at the site of lesion. We first treated SDF1 in the culture medium and observed a dramatic change in nuclear localization (Fig. 4A, 4B). SDF1 treatment significantly weakened the 
A

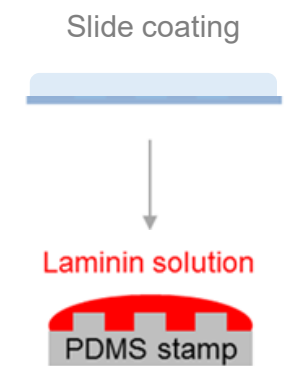

Air
washing
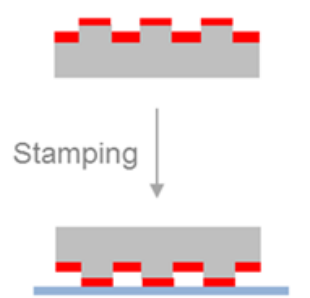

Stem cell seeding

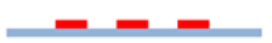

B

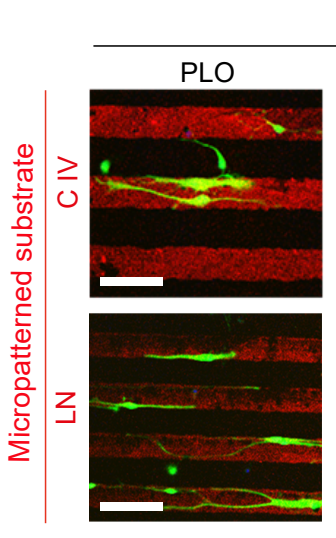

C

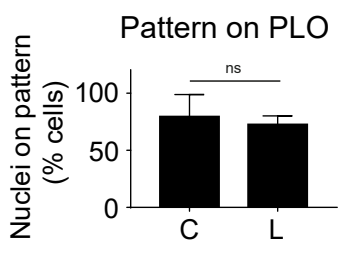

Coated substrate
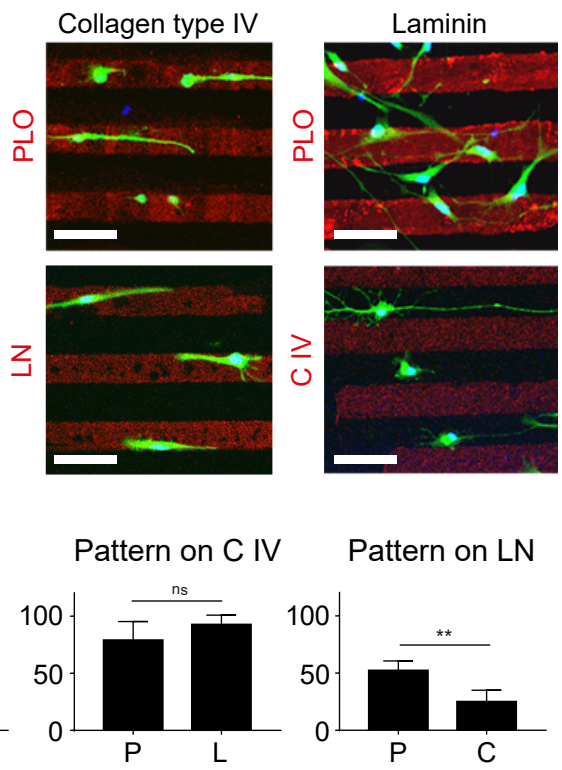

\section{Pattern on LN}

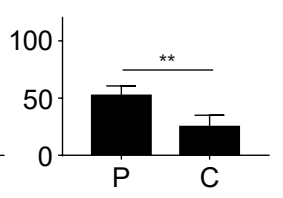

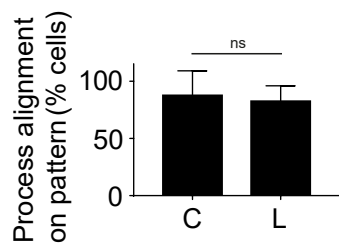
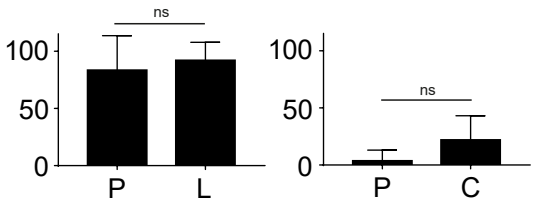

Fig. 3. ECM preference of adult NS/PCs using cell micropatterning. (A) Schematic diagram of the micropattern assay. One substrate was applied on the coverglass, and another substrate was loaded on a PDMS microstamp and stamped onto the coated coverglass. (B) Representative images of cell micropatterning using collagen type IV (C IV), laminin (LN), and PLO (P) as control. SVZ-derived progenitors preferred to adhere to the patterned substrate, with the exception of cells on the laminin-coated coverglass. Cells exhibited stronger adherence to laminin over collagen type IV, and the addition of collagen type IV on laminin decreased the adhesive effect of laminin (right). Scale bar, $50 \mu \mathrm{m}$. (C, D) The percentage of cells attached to patterned or coated substrates determined by the positioning of their nuclei and process alignment on the micropatterned area. Data represent mean and SD from at least three independent experiments. ${ }^{* *} \mathrm{p}<0.01, \mathrm{n} . \mathrm{s},>0.05$. ECM, extracellular matrix; PDMS, polydimethylsiloxane; PLO, polyornitine; SVZ, subventricular zone; SD, standard deviation.

inhibitory effect of collagen type IV on laminin preference; thus, most SVZ-derived progenitors were localized on the pattern. The vascular ECM components partly share integrin receptors: the laminin receptors are integrin $\alpha 3 \beta 1, \alpha 3 \beta 6$, and $\alpha 6 \beta 4$, while the collagen receptors are $\alpha 1 \beta 1, \alpha 2 \beta 1$ [40]. We then pre-incubated adult NS/PCs with a GoH3 antibody, also known as a6 integrin antibody, to selectively block laminin signaling. Functional blocking of a6 integrin perturbs the effect of SDF1 on the ECM preference of SVZ-derived progenitors. Thus, $\alpha 6$ integrin blockage may have reduced the adhesive force of combined ECM molecules, which resulted in an adhesive capacity similar to that of collagen type IV. A previous study has also shown that blockage of a 6 integrin perturbs homing to the laminin-rich basal lamina of vessels [24]. Collectively, these data indicate that the ECM preference of adult NS/ PCs can be changed by chemokines and specific integrin receptor expression.

\section{DISCUSSION}

Within the SVZ of adult mouse brain, NSCs and progenitor cells contact the basal lamina of blood vessels at one end and the ependymal cell layer lining the ventricles at the other end [7,22, 24]. Often specialized ECM structures termed fractones provide the anchoring spots for the apical end of NSCs $[21,25]$. Thus, we explored the distribution of major ECM components in two major areas, the basal lamina and fractones.

The ECM composition of the basal lamina is distinctly specialized in the SVZ. In contrast to the fairly homogenous ECM composition of cerebral blood vessels, the vasculature in the SVZ exhibits a distinct distribution of vascular ECM proteins such as laminin and collagen type IV. In the neurogenic niche, vessels express large amounts of laminin compared with collagen type IV, whereas cortical blood vessels are surrounded by an equivalent 

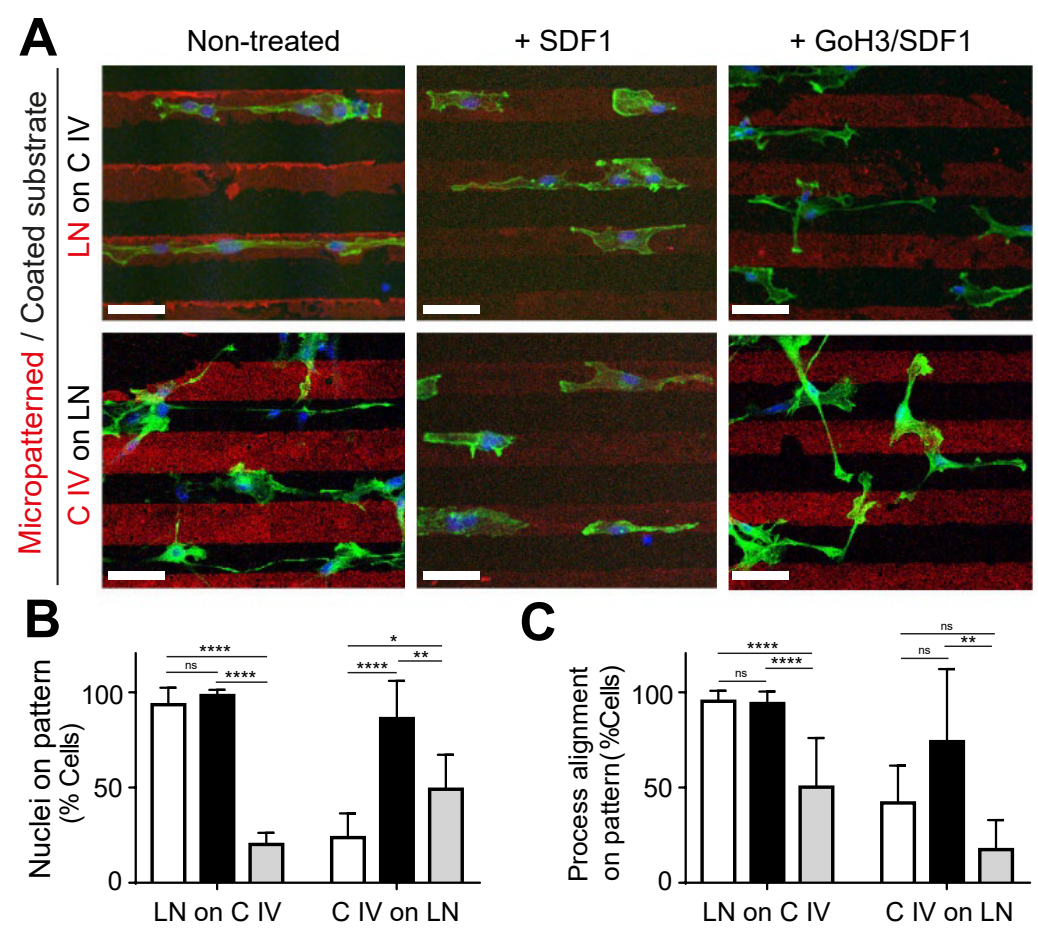

Fig. 4. Perturbation of ECM preference by SDF1 treatment and functional blocking of a 6 integrin. (A) Representative images of cell micropatterning. SVZ-derived progenitors were seeded on laminin pattern on collagen type IV coating (LN on C IV; upper panel) and collagen type IV pattern on laminin coating (C IV on LN; lower panel). SDF1 was added to the cultured medium, and cells were pretreated with GoH3 (blocking antibody for a6 integrin) prior to the micropattern assay. SDF1 eliminated the inhibitory effects of collagen type IV on laminin, whereas GoH treatment perturbed the laminin preference of adult NS/PCs. Scale bar, $50 \mu \mathrm{m}$. (B,C) The percentage of cells attached to patterned or coated substrates determined by nuclei positioning and process alignment. Data represent mean and SD from at least three independent experiments. ${ }^{*} \mathrm{p}<0.05,{ }^{* *} \mathrm{p}<0.01,{ }^{* * *} \mathrm{p}<0.0001, \mathrm{n} . \mathrm{s},>0.05$. ECM, extracellular matrix; SVZ, subventricular zone; SDF1; stromal-derived factor 1; NS/PCs, neural stem/progenitor cells; SD, standard deviation.

amount of laminin and collagen type IV. There have been no previous reports on the association between ECM and vessel size in the brain, but a few studies have found some correlations in pathological conditions. During vascular remodeling in type 2 diabetic rats, collagen deposition resulted in a two-fold increase in the wallto-lumen ratio of the middle cerebral arteries, possibly increasing the risk of cerebrovascular disease and stroke [41]. In collagen VInull mice, the width of the basal lamina was reduced by half due to poor deposition of collagen type IV and laminin [42]. Changes in the assembly of the vascular basal lamina resulted in reduced tumor progression. Thus, we speculated that assembly of the basal lamina is critical for the maintenance of adult NSCs and that different ECM compositions play a role in blood vessel-associated attachment and migration of NS/PCs in the SVZ.

We have also captured the development of laminin-rich fractones on the ependymal layer of the lateral ventricle during brain development. Fractones were visualized as small puncta during the embryonic stage. They form branching patterns during the postnatal period and gradually attain a relatively uniform, speckled shape. Although fractones were initially thought to be derived from the vascular basal lamina, more recent studies have suggested that they are produced by ependymal cells and/or GFAP-positive NSCs in the SVZ [24-26]. Our current observations also favor the ependymal origin of fractones. In addition, we found that the formation and relocalization of speckle-shaped fractones at the border of ependymal cells were temporally associated with the emergence of motile cilia in ependymal cells. Thus, we speculate that cilia play significant roles in fraction development, and further exploration is required to clarify this issue.

To monitor the influence of different compositions of ECM, we subtracted other factors, such as endothelial cells and pericytes that exist in the vascular niche. Since a large number of studies have validated the effectiveness of the micropattern method to mimic in vivo conditions, we used different substrates for coating and micropatterning to distinguish the adhesive effect of each ECM protein. Modification of substrate composition and topography has been reported to produce dramatic effects on neural progenitor alignment and cell morphology $[37,38]$. For example, micropatterned substrates restricted neurite extension by aligning neurites with the directionality of grooves and elevations, forming 
parallel tracts of Tuj1+ neurons. Moreover, surface topography has been shown to affect fibroblast migration [43]. Using the micropattern method, we found that SVZ-derived progenitors exhibited increased adhesiveness to laminin compared to collagen type IV. Micropatterned collagen type IV inhibits cell adhesion to the laminin-coated surface. Thus, our findings might explain the relative spatial proximity between neuronal progenitors and lamininintensive blood vessels.

We observed that the composition of the vascular basal lamina varies within the SVZ, thereby affecting the adhesion of adult NS/ PCs. Alterations in the expression of vascular basal lamina have been well established in ischemic stroke models [44]. Following middle cerebral artery occlusion, there is a significant but gradual local decrease in the microvascular expression or integrity of the major ECM components laminin-1 and -5, collagen type IV, and fibronectin $[45,46]$. Alterations in the expression of vascular basal lamina can be explained by local proteolysis initiated by focal ischemia, which is associated with reduced mechanical stress. Furthermore, integrins play a key role in cell adhesion to different ECM compositions, and integrin subunits are distributed in the microvasculature in distinct expression patterns [47]. Integrin subunits $\alpha 1$ and $\alpha 6$ are distributed throughout the cerebral capillaries in a pattern identical to that of subunit $\beta 1$ on the endothelium and its ligands laminin, collagen type IV, and fibronectin. In focal ischemia, reduced expression occurs within 1 to 2 hours in a specific region, which is accompanied by changes in ECM expression of the basal lamina. Although the regulation of integrin expression associated with changes in ECM composition is not well understood, integrins may act as both attractants and repellents depending on the differential composition of ECM in the basal lamina. Our findings might therefore be key to generating NS/PC-specific branched vasculature, potentially providing a scaffold for transplanted cells or vascular grafts.

\section{ACKNOWLEDGEMENTS}

This research was supported by the Brain Research Program and Bio \& Medical Technology Development Program of the National Research Foundation of Korea (NRF) funded by the Ministry of Science \& ICT (NRF-2015M3C7A1028790, NRF2017M3A9B3061308). This research was also supported by Basic Science Research Program through the National Research Foundation of Korea (NRF) funded by the Ministry of Education (NRF-2016R1D1A1A02937581, NRF-2018R1D1A1A02086190).

\section{REFERENCES}

1. Lois C, Alvarez-Buylla A (1993) Proliferating subventricular zone cells in the adult mammalian forebrain can differentiate into neurons and glia. Proc Natl Acad Sci U S A 90:20742077.

2. Palmer TD, Takahashi J, Gage FH (1997) The adult rat hippocampus contains primordial neural stem cells. Mol Cell Neurosci 8:389-404.

3. Reynolds BA, Weiss S (1992) Generation of neurons and astrocytes from isolated cells of the adult mammalian central nervous system. Science 255:1707-1710.

4. Kim WR, Chun SK, Kim TW, Kim H, Ono K, Takebayashi H, Ikenaka K, Oppenheim RW, Sun W (2011) Evidence for the spontaneous production but massive programmed cell death of new neurons in the subcallosal zone of the postnatal mouse brain. Eur J Neurosci 33:599-611.

5. Kim JY, Choi K, Shaker MR, Lee JH, Lee B, Lee E, Park JY, Lim MS, Park CH, Shin KS, Kim H, Geum D, Sun W (2016) Promotion of cortical neurogenesis from the neural stem cells in the adult mouse subcallosal zone. Stem Cells 34:888-901.

6. Palmer TD, Willhoite AR, Gage FH (2000) Vascular niche for adult hippocampal neurogenesis. J Comp Neurol 425:479494.

7. Tavazoie M, Van der Veken L, Silva-Vargas V, Louissaint M, Colonna L, Zaidi B, Garcia-Verdugo JM, Doetsch F (2008) A specialized vascular niche for adult neural stem cells. Cell Stem Cell 3:279-288.

8. Licht T, Keshet E (2015) The vascular niche in adult neurogenesis. Mech Dev 138 Pt 1:56-62.

9. Lacar B, Young SZ, Platel JC, Bordey A (2011) Gap junctionmediated calcium waves define communication networks among murine postnatal neural progenitor cells. Eur J Neurosci 34:1895-1905.

10. Bovetti S, Hsieh YC, Bovolin P, Perroteau I, Kazunori T, Puche AC (2007) Blood vessels form a scaffold for neuroblast migration in the adult olfactory bulb. J Neurosci 27:5976-5980.

11. Whitman MC, Fan W, Rela L, Rodriguez-Gil DJ, Greer CA (2009) Blood vessels form a migratory scaffold in the rostral migratory stream. J Comp Neurol 516:94-104.

12. Ohab JJ, Fleming S, Blesch A, Carmichael ST (2006) A neurovascular niche for neurogenesis after stroke. J Neurosci 26:13007-13016.

13. Thored P, Wood J, Arvidsson A, Cammenga J, Kokaia Z, Lindvall O (2007) Long-term neuroblast migration along blood vessels in an area with transient angiogenesis and increased vascularization after stroke. Stroke 38:3032-3039. 
14. Kojima T, Hirota Y, Ema M, Takahashi S, Miyoshi I, Okano H, Sawamoto K (2010) Subventricular zone-derived neural progenitor cells migrate along a blood vessel scaffold toward the post-stroke striatum. Stem Cells 28:545-554.

15. Villeda SA, Luo J, Mosher KI, Zou B, Britschgi M, Bieri G, Stan TM, Fainberg N, Ding Z, Eggel A, Lucin KM, Czirr E, Park JS, Couillard-Després S, Aigner L, Li G, Peskind ER, Kaye JA, Quinn JF, Galasko DR, Xie XS, Rando TA, Wyss-Coray T (2011) The ageing systemic milieu negatively regulates neurogenesis and cognitive function. Nature 477:90-94.

16. Karakatsani A, Shah B, Ruiz de Almodovar C (2019) Blood vessels as regulators of neural stem cell properties. Front Mol Neurosci 12:85.

17. Temple S (2001) The development of neural stem cells. Nature 414:112-117.

18. Shingo T, Sorokan ST, Shimazaki T, Weiss S (2001) Erythropoietin regulates the in vitro and in vivo production of neuronal progenitors by mammalian forebrain neural stem cells. J Neurosci 21:9733-9743.

19. Hynes RO (2009) The extracellular matrix: not just pretty fibrils. Science 326:1216-1219.

20. Kerever A, Schnack J, Vellinga D, Ichikawa N, Moon C, Arikawa-Hirasawa E, Efird JT, Mercier F (2007) Novel extracellular matrix structures in the neural stem cell niche capture the neurogenic factor fibroblast growth factor 2 from the extracellular milieu. Stem Cells 25:2146-2157.

21. Mercier F (2016) Fractones: extracellular matrix niche controlling stem cell fate and growth factor activity in the brain in health and disease. Cell Mol Life Sci 73:4661-4674.

22. Kerever A, Mercier F, Nonaka R, de Vega S, Oda Y, Zalc B, Okada Y, Hattori N, Yamada Y, Arikawa-Hirasawa E (2014) Perlecan is required for FGF-2 signaling in the neural stem cell niche. Stem Cell Res 12:492-505.

23. Douet V, Kerever A, Arikawa-Hirasawa E, Mercier F (2013) Fractone-heparan sulphates mediate FGF-2 stimulation of cell proliferation in the adult subventricular zone. Cell Prolif 46:137-145.

24. Shen Q, Wang Y, Kokovay E, Lin G, Chuang SM, Goderie SK, Roysam B, Temple S (2008) Adult SVZ stem cells lie in a vascular niche: a quantitative analysis of niche cell-cell interactions. Cell Stem Cell 3:289-300.

25. Nascimento MA, Sorokin L, Coelho-Sampaio T (2018) Fractone bulbs derive from ependymal cells and their laminin composition influence the stem cell niche in the subventricular zone. J Neurosci 38:3880-3889.

26. Sato Y, Kiyozumi D, Futaki S, Nakano I, Shimono C, Kaneko N, Ikawa M, Okabe M, Sawamoto K, Sekiguchi K (2019) Ven- tricular-subventricular zone fractones are speckled basement membranes that function as a neural stem cell niche. Mol Biol Cell 30:56-68.

27. Ihrie RA, Alvarez-Buylla A (2011) Lake-front property: a unique germinal niche by the lateral ventricles of the adult brain. Neuron 70:674-686.

28. Sanes JR (1989) Extracellular matrix molecules that influence neural development. Annu Rev Neurosci 12:491-516.

29. Baktash Y, Madhav A, Coller KE, Randall G (2018) Single particle imaging of polarized hepatoma organoids upon hepatitis $C$ virus infection reveals an ordered and sequential entry process. Cell Host Microbe 23:382-394.e5.

30. Hamann GF, Liebetrau M, Martens H, Burggraf D, Kloss CU, Bültemeier G, Wunderlich N, Jäger G, Pfefferkorn T (2002) Microvascular basal lamina injury after experimental focal cerebral ischemia and reperfusion in the rat. J Cereb Blood Flow Metab 22:526-533

31. Leone TC, Lehman JJ, Finck BN, Schaeffer PJ, Wende AR, Boudina S, Courtois M, Wozniak DF, Sambandam N, BernalMizrachi C, Chen Z, Holloszy JO, Medeiros DM, Schmidt RE, Saffitz JE, Abel ED, Semenkovich CF, Kelly DP (2005) PGClalpha deficiency causes multi-system energy metabolic derangements: muscle dysfunction, abnormal weight control and hepatic steatosis. PLoS Biol 3:e101.

32. Kazanis I, Lathia JD, Vadakkan TJ, Raborn E, Wan R, Mughal MR, Eckley DM, Sasaki T, Patton B, Mattson MP, Hirschi KK, Dickinson ME, ffrench-Constant C (2010) Quiescence and activation of stem and precursor cell populations in the subependymal zone of the mammalian brain are associated with distinct cellular and extracellular matrix signals. J Neurosci 30:9771-9781.

33. Mirzadeh Z, Doetsch F, Sawamoto K, Wichterle H, AlvarezBuylla A (2010) The subventricular zone en-face: wholemount staining and ependymal flow. J Vis Exp 39:1938.

34. Lee E, Choi J, Jo Y, Kim JY, Jang YJ, Lee HM, Kim SY, Lee HJ, Cho K, Jung N, Hur EM, Jeong SJ, Moon C, Choe Y, Rhyu IJ, Kim H, Sun W (2016) ACT-PRESTO: rapid and consistent tissue clearing and labeling method for 3-dimensional (3D) imaging. Sci Rep 6:18631.

35. Joo S, Yeon Kim J, Lee E, Hong N, Sun W, Nam Y (2015) Effects of ECM protein micropatterns on the migration and differentiation of adult neural stem cells. Sci Rep 5:13043.

36. Kerever A, Yamada T, Suzuki Y, Mercier F, Arikawa-Hirasawa E (2015) Fractone aging in the subventricular zone of the lateral ventricle. J Chem Neuroanat 66-67:52-60.

37. Curtis A, Wilkinson C (1997) Topographical control of cells. Biomaterials 18:1573-1583. 
38. Recknor JB, Sakaguchi DS, Mallapragada SK (2006) Directed growth and selective differentiation of neural progenitor cells on micropatterned polymer substrates. Biomaterials 27:40984108.

39. Kokovay E, Goderie S, Wang Y, Lotz S, Lin G, Sun Y, Roysam B, Shen Q, Temple S (2010) Adult SVZ lineage cells home to and leave the vascular niche via differential responses to SDF1/ CXCR4 signaling. Cell Stem Cell 7:163-173.

40. Hynes RO (2002) Integrins: bidirectional, allosteric signaling machines. Cell 110:673-687.

41. Harris AK, Hutchinson JR, Sachidanandam K, Johnson MH, Dorrance AM, Stepp DW, Fagan SC, Ergul A (2005) Type 2 diabetes causes remodeling of cerebrovasculature via differential regulation of matrix metalloproteinases and collagen synthesis: role of endothelin-1. Diabetes 54:2638-2644.

42. You WK, Bonaldo P, Stallcup WB (2012) Collagen VI ablation retards brain tumor progression due to deficits in assembly of the vascular basal lamina. Am J Pathol 180:1145-1158.
43. Kaiser JP, Reinmann A, Bruinink A (2006) The effect of topographic characteristics on cell migration velocity. Biomaterials 27:5230-5241.

44. del Zoppo GJ, Mabuchi T (2003) Cerebral microvessel responses to focal ischemia. J Cereb Blood Flow Metab 23:879894.

45. Hamann GF, Okada Y, del Zoppo GJ (1996) Hemorrhagic transformation and microvascular integrity during focal cerebral ischemia/reperfusion. J Cereb Blood Flow Metab 16:1373-1378.

46. Hamann GF, Okada Y, Fitridge R, del Zoppo GJ (1995) Microvascular basal lamina antigens disappear during cerebral ischemia and reperfusion. Stroke 26:2120-2126.

47. Haring HP, Akamine BS, Habermann R, Koziol JA, Del Zoppo GJ (1996) Distribution of integrin-like immunoreactivity on primate brain microvasculature. J Neuropathol Exp Neurol 55:236-245. 